\title{
Tunneling decay of self-gravitating vortices
}

\author{
Éric Dupuis ${ }^{1, \star}$, Yan Gobeil ${ }^{1,2, \star \star}$, Bum-Hoon Lee Le, $^{3, \star \star \star}$, Wonwoo Lee Le $^{3, \star \star \star \star}$, Richard \\ MacKenzie $^{1, \dagger}$, Manu B. Paranjape ${ }^{1, \ddagger}$, Urjit A. Yajnik ${ }^{5, \S}$, and Dong-han Yeom ${ }^{6,7, \pi}$ \\ ${ }^{1}$ Groupe de Physique des Particules, Département de physique, Université de Montréal, \\ C. P. 6128, Succursale Centre-ville, Montréal, Québec, Canada H3C 3J7 \\ ${ }^{2}$ Department of Physics, Ernest Rutherford Physics Building, McGill University, 3600 rue University, \\ Montréal, Québec, Canada H3A 2T8 \\ ${ }^{3}$ Center for Quantum Spacetime, Sogang University, Seoul 04107, Korea \\ ${ }^{4}$ Department of Physics, Sogang University, Seoul 04107, Korea \\ ${ }^{5}$ Department of Physics, Indian Institute of Technology Bombay, Mumbai 400076, India \\ ${ }^{6}$ Leung Center for Cosmology and Particle Astrophysics, National Taiwan University, Taipei 10617, Taiwan \\ ${ }^{7}$ Asia Pacific Center for Theoretical Physics, and Department of Physics, Pohang University of Science and \\ Technology, Pohang 37673, Korea
}

\begin{abstract}
We investigate tunneling decay of false vortices in the presence of gravity, in which vortices are trapped in the false vacuum of a theory of scalar electrodynamics in three dimensions. The core of the vortex contains magnetic flux in the true vacuum, while outside the vortex is the appropriate topologically nontrivial false vacuum. We numerically obtain vortex solutions which are classically stable; however, they could decay via tunneling. To show this phenomenon, we construct the proper junction conditions in curved spacetime. We find that the tunneling exponent for the vortices is half that for Coleman-de Luccia bubbles and discuss possible future applications.
\end{abstract}

\section{Brief summary of the talk}

Gauged vortices are topological soliton solutions in the theory of a complex scalar field $\phi$ with $U$ (1) symmetry in three spacetime dimensions. For standard vortices, the potential is minimized for a nonzero value of $|\phi|$, so the vacuum manifold is a circle, as is spatial infinity. In a finite-energy field configuration, the field $\phi$ must tend towards a vacuum value at spatial infinity and gauge field is necessary. The phase of the field $\phi$ changes by $2 \pi n$, where the integer $n$ is the winding number of the configuration representing topologically distinct classes. The energy of the vortex solution consists of three parts as follows: The contribution from the magnetic flux stabilizes the configuration against collapse, while the contribution from the potential stabilizes the configuration against expansion. The

\footnotetext{
$\star$ e-mail: eric.dupuis.1@umontreal.ca

$\star \star$ e-mail: yan.gobeil@mail.mcgill.ca

$\star \star \star$ e-mail: bhl@sogang.ac.kr

$\star \star \star \star$ e-mail: warrior@ sogang.ac.kr ${ }^{*}$

$\dagger$ e-mail: richard.mackenzie@umontreal.ca

‡e-mail: paranj@lps.umontreal.ca

$\S_{\text {e-mail: yajnik@iitb.ac.in }}$

Ile-mail: innocent.yeom@gmail.com
} 


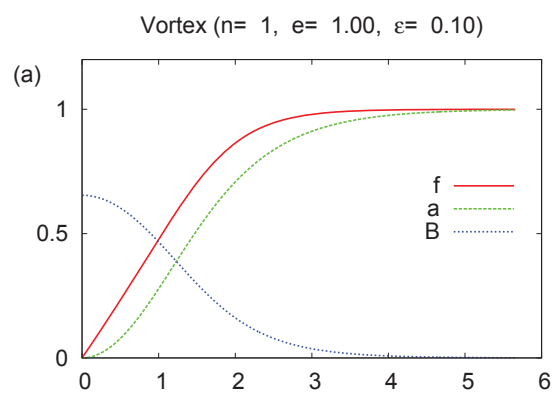

(a)

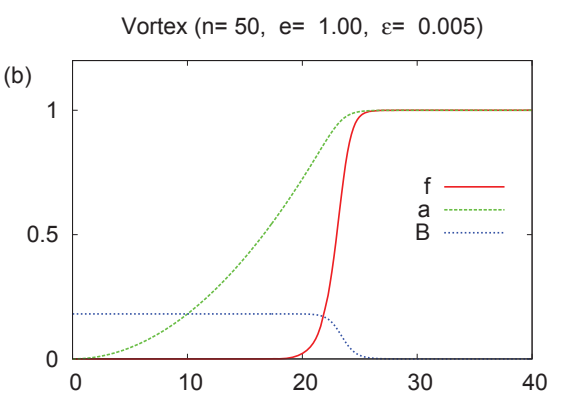

(b)

Figure 1. Vortex profile for (a) thick-wall and (b) thin-wall vortices. Displayed are the functions $f(r)$ and $a(r)$ and the magnetic field $B(r)=n a^{\prime}(r) / e r$. (a) Vortex $(n=1, e=1.00, \varepsilon=0.10)$ and (b) Vortex $(n=50, e=1.00$, $\varepsilon=0.005)$. For more details, see Ref. [7].

covariant derivative of the scalar field also contributes an energy as a linear function of the radius. The profile of the scalar field can sometimes be flat inside and outside a thin transition region between these two values; we call such configurations thin-wall vortices. In this case, an approximate analytical treatment known as the thin-wall approximation can be used.

In such a model in four spacetime dimensions, the soliton becomes an one-dimensional topological defect $[1,2]$. The configuration can be infinite or form a closed loop. In the cosmological context they could appear as cosmic strings [3, 4], possibly playing a role in structure formation or having other observable effects $[5,6]$.

In earlier work [7], we studied the decay of vortices trapped in the symmetry-breaking false vacuum, in which the scalar field interpolates between a true vacuum state at the origin and a false vacuum state at spatial infinity. We chose the vacuum energy in the symmetry-restoring true vacuum to be negative, while that in the false vacuum vanishes. We obtained the numerical solutions representing both thick and thin vortices as shown in Figure 1. Thin-wall vortices require a large winding number and a small difference, $\varepsilon$, between the false and true vacuum energies. For thin-wall vortices, if the false vacuum contains a metastable vortex solution instead of the homogeneous configuration at the initial moment, the vortex was classically stable at a given radius. However, through quantum tunneling, the vortex could become a larger one and expand leaving behind it the symmetric true vacuum. It is possible that conventional vacuum decay proceeds by tunneling and is exponentially suppressed, while vortex tunneling is unsuppressed or is only slightly suppressed. In this case, the presence of vortices in the universe would catalyze vacuum decay. This occurs as one approaches the dissociation limit, in which the tunneling barrier between a classically stable vortex and an unstable, expanding vortex shrinks away. Thus, there is a range of parameter space for which vortices are classically stable but trigger the decay of the false vacuum in an essentially unsuppressed manner.

As extension of the work [8], we studied the decay of cosmic strings that are trapped in the false vacuum in a theory of scalar electrodynamics in four spacetime dimensions. We restricted our analysis to the case of thin-walled cosmic strings which occur when large magnetic flux is trapped inside the string. Thus the string looks like a tube of fixed radius, at which it is classically stable. The core of the string contains magnetic flux in the true vacuum, while outside the string, separated by a thin wall, is the false vacuum. The string decays by tunneling to a configuration which is represented by a bulge as shown in Figure 2, where the region of true vacuum within is ostensibly enlarged. The bulge 


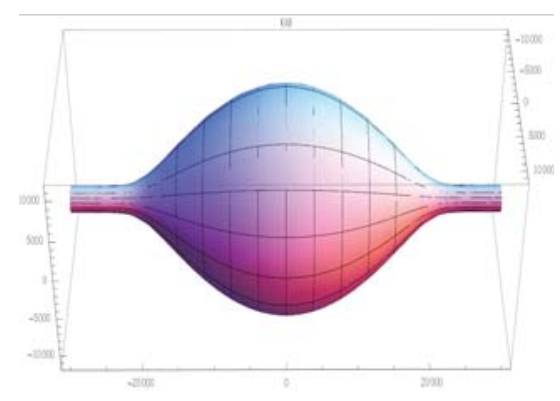

(a)

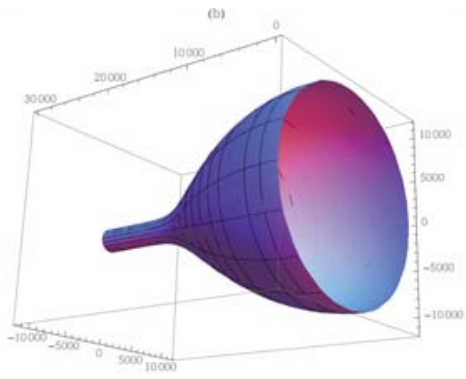

(b)

Figure 2. (a) Cosmic string profile at the bounce point. (b) Cut away of the cosmic string profile at bounce point. For more details, see Ref. [8].

can be described as the meeting of a kink soliton-antisoliton pair along the length of the string. It can be described as a bulge appearing in the initial string, starting from one of small, classically stable radius, expanding to a fat string of large, classically unstable (to expansion) radius and then returning back to one of small radius along its length. This configuration is the bounce point of a corresponding $O$ (2)-symmetric instanton, which we can determine numerically. Once the bulge appears it expands rapidly in real time. The paired soliton and antisoliton recede from each other along the length of the string with a velocity that quickly approaches the speed of light, leaving behind a fat tube. At the same time the radius of the fat tube that is being formed expands (transversely) as it is no longer classically stable, converting false vacuum to the true vacuum with ever-diluting magnetic field within. The rate of this expansion is determined by the energy difference between the true vacuum and the false vacuum. Our analysis could be applied to a network of cosmic strings formed in the very early Universe or vortex lines in a superheated superconductor.

In the present work [9], we are studying the effect of vortices on the tunneling decay of a symmetry-breaking false vacuum in three spacetime dimensions with gravity. We derive the equations of motion for the gauge, scalar field, and metric functions and impose the appropriate boundary conditions to get the finite energy configuration. We present numerical solutions for both thick- and thin-wall vortices. The numerical solutions have inside true vacuum state and the magnetic flux with the wall in the presence of gravity. By varying the difference between the false and true vacuum states, we investigate how the existence of the solution can be affected by the strength of $\kappa=8 \pi G$. The existence of static vortex solutions with gravity is established. After that, we investigate more closely the metastability of thin-wall vortices. For this purpose, we employ the Israel junction conditions [10] to understand if the vortex can tunnel through a certain potential barrier and expand dynamically. In the framework of junction conditions, the wall is considered to be a surface layer in the sense that a nonvanishing surface energy density $\sigma$ exists. This implies a modification of the spacetime. The exterior spacetime with a vortex confined within a finite radius is locally Minkowski with a conical defect [11]. The conical defect is the analog of the Schwarzschild mass parameter that is familiar in the $3+1$ dimensional context.

Figure 3 shows the causal structure of thin-wall vortices, in which the yellow region is inside the wall and the green region is outside the wall. Left: as the vortex tunnels toward a collapsing phase, it becomes a timelike cusp (that behaves like a massive particle) which is located at $r=0$. Right: as 

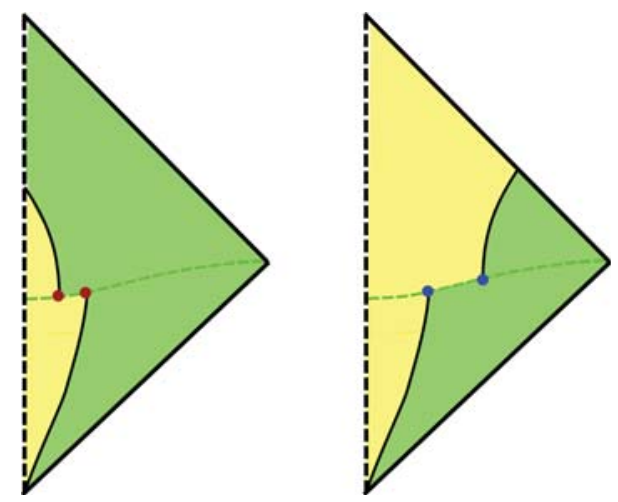

Figure 3. The causal structure of thin-wall vortices.

the vortex tunnels toward an expanding phase, the radius increases to infinity. We are interested in the right case.

If the scalar field is in a metastable vacuum state, the tunneling process from the false vacuum state to the true vacuum state can occur via potential barrier penetration, which is the nucleation process of a vacuum bubble $[12,13]$. In the semiclassical approximation, the decay rate of the metastable vacuum state per unit time per unit volume is given by

$$
\Gamma / V=A e^{-B}[1+O(\hbar)]
$$

where the coefficient $A$ comes from the determinant arising in the saddle-point evaluation of the path integral and the exponent $B$ is the difference between the Euclidean action of the bounce solution and the action of the background solution, i.e. $B=S_{\text {bounce }}^{E}-S_{\text {bckg }}^{E}$. The determinant factor must exclude the integration over the zero modes. The Coleman-de Luccia (CdL) bounce has translation invariance in all direction, giving three zero modes. The vortex only has time translation invariance, giving one zero mode. The position of the vortex is fixed once and for all. These modes are removed. Instead, the corresponding degrees of freedom (either the center of the vacuum bounce in spacetime or the center of the vortex bounce in time) are integrated over. We are interested in finding the exponent $B$. In the presence of a vortex, a similar process exists. As can be deduced in the collective coordinate approximation suggested in [9], quantum fluctuations of the vortex radius can also lead to a phase transition. In this case, the relevant instanton describes the expansion of the wall. To understand the cosmological relevance of the vortex, we compare the false vacuum lifetime in presence and absence of a vortex.

For the decay probability, we can consider a variety of decay channels, i.e. CdL bounce, oscillating bounce, Hawking-Moss instanton, tunneling vortices, etc. The remarkable thing about the decay probability is that the channel for the tunneling vortex can be more dominant than that for the CdL bounce. Computations in the thin-wall limit have shown that the false vortex's contribution to metastability remains dominant, and more so as $\varepsilon \rightarrow 0$ limit. Another effect is the suppression of tunneling when the vacuum energy density reaches a limiting value, $\varepsilon \rightarrow \varepsilon_{c}$. This critical value is increased as gravity is turned on, but only very slightly for reasonable values of $\kappa$. In this respect, this is another minor way in which gravity stabilizes solutions of this model.

The extension to the decay of the string with gravity could be interesting. We postpone to discuss this issue in future works. 


\section{Acknowledgements}

We would like to thank Sung-Won Kim, Remo Ruffini, Sang-Pyo Kim, Gungwon Kang, Jonghyuk Yoon, Hyung Won Lee, and Hyeong-Chan Kim for their hospitality at Joint Conference of 13th International Conference on Gravitation, Astrophysics, and Cosmology, and 15th Italian-Korean Symposium on Relativistic Astrophysics in Korea, 03-07 Jul 2017. This work was financially supported in part by the Natural Sciences and Engineering Research Council of Canada and the Fonds de recherche du Québec - Nature et technologies. RM, MP and UY thank the Ministre des Relations internationales et de la Francophonie du Gouvernement du Québec under the Cooperation QuébecMaharashtra for continuing financial support. BHL is supported by Basic Science Research Program through the National Research Foundation of Korea (NRF-2017R1D1A1B03028310). WL is supported by Basic Science Research Program through the National Research Foundation of Korea (2016R1D1A1B01010234). DY is supported by the Leung Center for Cosmology and Particle Astrophysics (LeCosPA) of National Taiwan University (103R4000).

\section{References}

[1] A. A. Abrikosov, Sov. Phys. JETP 5, 1174 (1957) [Zh. Eksp. Teor. Fiz. 32, 1442 (1957)].

[2] H. B. Nielsen and P. Olesen, Nucl. Phys. B 61, 45 (1973).

[3] T. W. B. Kibble, J. Phys. A 9, 1387 (1976).

[4] T. W. B. Kibble, Phys. Rept. 67, 183 (1980).

[5] R. H. Brandenberger, Nucl. Phys. Proc. Suppl. 246-247, 45 (2014).

[6] H. Tashiro, T. Sekiguchi and J. Silk, JCAP 1401, 013 (2014).

[7] B.-H. Lee, W. Lee, R. MacKenzie, M. B. Paranjape, U. A. Yajnik and D.-h. Yeom, Phys. Rev. D 88, 085031 (2013).

[8] B.-H. Lee, W. Lee, R. MacKenzie, M. B. Paranjape, U. A. Yajnik and D.-h. Yeom, Phys. Rev. D 88, 105008 (2013).

[9] É. Dupuis, Y. Gobeil, B.-H. Lee, W. Lee, R. MacKenzie, M. B. Paranjape, U. A. Yajnik and D.-h. Yeom, arXiv:1709.03839 [hep-th].

[10] W. Israel, Nuovo Cim. B 44S10, 1 (1966) [Nuovo Cim. B 44, 1 (1966)] (erratum: Nuovo Cim. B 48, 463 (1967)).

[11] S. Deser, R. Jackiw and G. 't Hooft, Annals Phys. 152, 220 (1984).

[12] S. R. Coleman, Phys. Rev. D 15, 2929 (1977) Erratum: [Phys. Rev. D 16, 1248 (1977)].

[13] C. G. Callan, Jr. and S. R. Coleman, Phys. Rev. D 16, 1762 (1977). 